\title{
Quasi-normality of idempotents on nilpotents
}

\author{
Tai Keun Kwak*1 (D), Seung Ick Lee ${ }^{2}$ (D), Yang Lee ${ }^{3,4}$ (D) \\ ${ }^{1}$ Daejin University, Department of Mathematics, Pocheon 11159, Korea \\ ${ }^{2}$ Pusan National University, Department of Mathematics, Pusan 46241, Korea \\ ${ }^{3}$ Yanbian University, Department of Mathematics, Yanji 133002, China \\ ${ }^{4}$ Daejin University, Institute of Basic Science, Pocheon 11159, Korea
}

\begin{abstract}
We study the structure of idempotents in non-Abelian rings, concerning a ring property near to the normality of idempotents on the set of nilpotents. We call a ring with such property right idempotent-quasi-normalizing on nilpotents (simply, right IQNN), and study the structure of right IQNN rings in relation with matrix rings, polynomial ring, and factor rings, by which we extend the class of right IQNN rings. It is proved that the class of IQNN rings contains the 2 by 2 full matrix rings over fields and the upper triangular matrix rings over reduced rings. It is shown that given any countable field $K$, there exists a semiprime IQNN algebra $R$ over $K$ such that the polynomial ring $R[x]$ over $R$ is IQNN but not NI, and the upper nilradical of $R[x]$ is zero.
\end{abstract}

Mathematics Subject Classification (2010). 16N40, 16U80

Keywords. right IQNN ring, idempotent, nilpotent, abelian ring, matrix ring, polynomial ring, NI ring, NR ring

\section{Introduction}

The structure of idempotents have been studied by many authors in various sorts of rings. A number of related results are obtained in the procedure of investigating the structures of polynomial rings and matrix rings. In this article we concentrate our attention on a property of idempotents on nilpotent elements that is similar to the normality, in non-Abelian rings. It is shown that the class of rings with such property is quite large.

Throughout this article every ring is associative with identity unless otherwise stated. Let $R$ be a ring. Denote the set of all idempotents in $R$ by $I(R)$ and write $I(R)^{\prime}=$ $I(R) \backslash\{0,1\}$. Let $J(R), N^{*}(R), N_{*}(R)$, and $N(R)$ denote the Jacobson radical, the upper nilradical, the lower nilradical, and the set of all nilpotent elements in $R$, respectively. We also use nilpotent for a nilpotent element for simplicity. Note that $N_{*}(R) \subseteq N^{*}(R) \subseteq N(R)$ and $N^{*}(R) \subseteq J(R) . \mathbb{Z}\left(\mathbb{Z}_{n}\right)$ denotes the ring of integers (modulo $n$ ). Denote the $n$ by $n$ full (resp., upper triangular) matrix ring over $R$ by $M_{a t}(R)$ (resp., $\left.T_{n}(R)\right)$. $E_{i j}$ denotes the matrix with $(i, j)$-entry 1 and elsewhere 0 , and write $D_{n}(R)=\left\{\left(a_{i j}\right) \in T_{n}(R) \mid a_{11}=\right.$ $\left.\cdots=a_{n n}=0\right\}$.

\footnotetext{
*Corresponding Author.

Email addresses: tkkwak@daejin.ac.kr (T.K. Kwak), maick@hanmail.net (S.I. Lee), ylee@pusan.ac.kr (Y. Lee)

Received: 19.03.2018; Accepted: 02.07.2018
} 


\section{Right idempotent-quasi-normalizing on nilpotents}

In this section, we introduce a new structure of idempotents in non-Abelian rings, concerning a ring property near to the normality of idempotents on the set of nilpotents.

Due to Feller [7], a ring is called right (resp., left) duo if every right (resp., left) ideal is an ideal; a ring is called duo if it is both right and left duo. It is obvious that a ring $R$ is right duo if and only if $R a \subseteq a R$ for all $a \in R$. A ring is usually called reduced if $N(R)=0$. A ring is usually called Abelian if every idempotent is central. Both one-sided duo rings and reduced rings are easily shown to be Abelian.

Lemma 2.1. For a ring $R$ with $I(R)^{\prime}$ nonempty, the following conditions are equivalent:

(1) $R$ is Abelian;

(2) Re is contained in $e R$ for all $e \in I(R)^{\prime}$;

(3) $e R$ is contained in Re for all $e \in I(R)^{\prime}$;

(4) $N(R) e$ is contained in $e N(R)$ for all $e \in I(R)^{\prime}$;

(5) $e N(R)$ is contained in $N(R)$ e for all $e \in I(R)^{\prime}$;

(6) $N(R) e$ is contained in $e R$ for all $e \in I(R)^{\prime}$;

(7) $e N(R)$ is contained in Re for all $e \in I(R)^{\prime}$.

Proof. (4) $\Rightarrow(1)$. Let $R$ be satisfy the condition (4), and assume on the contrary that there exist $r \in R$ and $e \in I(R)$ such that $\operatorname{er}(1-e) \neq 0$. Note $\operatorname{er}(1-e) \in N(R)$ and $e, 1-e \in I(R)^{\prime}$.

By the condition $(4), \operatorname{er}(1-e)=[\operatorname{er}(1-e)](1-e)=(1-e) s$ for some $s \in N(R)$. This yields

$$
0=(1-e)[e r(1-e)]=(1-e)[(1-e) s]=(1-e) s \neq 0,
$$

a contradiction. Thus $R$ is Abelian. The proofs of $(2) \Rightarrow(1),(3) \Rightarrow(1),(5) \Rightarrow(1),(6) \Rightarrow$ $(1)$, and $(7) \Rightarrow(1)$ are similar. Other directions are obvious.

We next consider a condition that is a proper generalization of the conditions (4) and (5) in Lemma 2.1.

Definition 2.2. A ring $R$ is said to be right idempotent-quasi-normalizing on nilpotents (simply, right $I Q N N)$ provided that $I(R)^{\prime}$ is empty, or else for every pair $(e, a) \in I(R)^{\prime} \times$ $N(R)$ there exists $(b, f) \in N(R) \times I(R)^{\prime}$ such that $e a=b f$. A left IQNN ring is defined symmetrically. A ring is $I Q N N$ if it is both right and left IQNN.

One-sided duo rings are Abelian, and Abelian rings are clearly right (left) IQNN. But right (left) IQNN rings need not be Abelian by the following examples.

Recall that the Cayley-Hamilton theorem in linear algebra states that every square matrix over a commutative ring satisfies its own characteristic equation. We will use this fact freely. The determinant (resp., trace) of $M$ is denoted by $\operatorname{det}(M)($ resp., $\operatorname{tr}(A))$, where $M$ is a given square matrix.

Lemma 2.3. Let $A$ be a commutative ring and $R=\operatorname{Mat}_{2}(A)$.

(1) $I(R)^{\prime}=\left\{\left(\begin{array}{cc}s & t \\ u & 1-s\end{array}\right) \in R \mid s(1-s)=t u\right\}$.

(2) Let $A$ be a commutative domain. Then $I(R)^{\prime}$ is the union of the following two sets:

$$
\left\{\left(\begin{array}{ll}
1 & 0 \\
0 & 0
\end{array}\right),\left(\begin{array}{ll}
0 & 0 \\
0 & 1
\end{array}\right),\left(\begin{array}{ll}
1 & t \\
0 & 0
\end{array}\right),\left(\begin{array}{ll}
1 & 0 \\
u & 0
\end{array}\right),\left(\begin{array}{ll}
0 & t \\
0 & 1
\end{array}\right),\left(\begin{array}{ll}
0 & 0 \\
u & 1
\end{array}\right) \mid t \neq 0, u \neq 0\right\}
$$

and

$$
\left\{\left(\begin{array}{cc}
s & t \\
u & 1-s
\end{array}\right) \mid s \notin\{0,1\} \text { and } s(1-s)=t u\right\} .
$$

(3) Let $A$ be a commutative domain. $N(R)$ is the union of the following two sets:

$$
\left\{\left(\begin{array}{ll}
0 & 0 \\
0 & 0
\end{array}\right),\left(\begin{array}{ll}
0 & t \\
0 & 0
\end{array}\right),\left(\begin{array}{ll}
0 & 0 \\
u & 0
\end{array}\right) \mid t \neq 0, u \neq 0\right\}
$$


and

$$
\left\{\left(\begin{array}{cc}
s & t \\
u & -s
\end{array}\right) \mid s \neq 0, t \neq 0, u \neq 0, \text { and } s^{2}=-t u\right\} .
$$

(4) Let $A$ be a filed. Then $I(R)^{\prime}$ is the union of the following two sets:

$$
\left\{\left(\begin{array}{ll}
1 & 0 \\
0 & 0
\end{array}\right),\left(\begin{array}{ll}
0 & 0 \\
0 & 1
\end{array}\right),\left(\begin{array}{ll}
1 & t \\
0 & 0
\end{array}\right),\left(\begin{array}{ll}
1 & 0 \\
u & 0
\end{array}\right),\left(\begin{array}{ll}
0 & t \\
0 & 1
\end{array}\right),\left(\begin{array}{ll}
0 & 0 \\
u & 1
\end{array}\right) \mid t \neq 0, u \neq 0\right\}
$$

and

$$
\left\{\left(\begin{array}{cc}
s & t \\
t^{-1} s(1-s) & 1-s
\end{array}\right)=\left(\begin{array}{cc}
s & t \\
{\left[t^{-1}(1-s)\right] s} & {\left[t^{-1}(1-s)\right] t}
\end{array}\right) \mid s \notin\{0,1\}, t \neq 0\right\} .
$$

(5) Let $A$ be a filed. Then $N(R)$ is the union of the following two sets:

$$
\left\{\left(\begin{array}{ll}
0 & 0 \\
0 & 0
\end{array}\right),\left(\begin{array}{cc}
0 & t \\
0 & 0
\end{array}\right),\left(\begin{array}{ll}
0 & 0 \\
u & 0
\end{array}\right) \mid t \neq 0, u \neq 0\right\}
$$

and

$$
\left\{\left(\begin{array}{cc}
s & t \\
-t^{-1} s^{2} & -s
\end{array}\right)=\left(\begin{array}{cc}
s & t \\
{\left[-t^{-1} s\right] s} & {\left[-t^{-1} s\right] t}
\end{array}\right) \mid s \neq 0, t \neq 0\right\}
$$

Proof. (1) Suppose that $E=\left(\begin{array}{ll}s & t \\ u & v\end{array}\right) \in I(R)^{\prime}$. Since $E^{2}=E, E$ satisfies the equation $x^{2}-x=0$. But $E \neq\left(\begin{array}{ll}0 & 0 \\ 0 & 0\end{array}\right),\left(\begin{array}{ll}1 & 0 \\ 0 & 1\end{array}\right)$ and so $x^{2}-x$ is the minimal polynomial of $E$. Hence $x^{2}-x$ is equal to the characteristic polynomial of $E$ by the Cayley-Hamilton theorem, entailing $\operatorname{tr}(E)=1$ and $\operatorname{det}(E)=0$. Hence $s+v=1$ and $s v=t u$. This yields $s(1-s)=t u$.

(2) Let $A$ be a commutative domain and suppose that $E=\left(\begin{array}{ll}s & t \\ u & v\end{array}\right) \in I(R)^{\prime}$. Then, by (1), $E=\left(\begin{array}{cc}s & t \\ u & 1-s\end{array}\right)$ with $s(1-s)=t u$.

Case 1. Suppose that $t=0$ and $u=0$.

Then $s^{2}=s$ and $v^{2}=v$ since $E^{2}=E$. So each of $s$ and $v$ is either 0 or 1 because $A$ is a domain. This implies that $E$ is either $\left(\begin{array}{ll}1 & 0 \\ 0 & 0\end{array}\right)$ or $\left(\begin{array}{ll}0 & 0 \\ 0 & 1\end{array}\right)$.

Case 2. Suppose that only one of $t$ and $u$ is nonzero.

Then $E$ is either $\left(\begin{array}{ll}s & t \\ 0 & v\end{array}\right)$ or $\left(\begin{array}{ll}s & 0 \\ u & v\end{array}\right)$. In each case, we have $s^{2}=s$ and $v^{2}=v$; hence each of $s$ and $v$ is either 0 or 1 because $A$ is a domain. Consequently $(s, v)$ is either $(1,0)$ or $(0,1)$ because $E \in I(R)^{\prime}$. Thus $E$ is one of the following:

$$
\left(\begin{array}{ll}
1 & t \\
0 & 0
\end{array}\right),\left(\begin{array}{ll}
1 & 0 \\
u & 0
\end{array}\right),\left(\begin{array}{ll}
0 & t \\
0 & 1
\end{array}\right), \text { and }\left(\begin{array}{ll}
0 & 0 \\
u & 1
\end{array}\right),
$$

where $0 \neq t, 0 \neq u$.

Case 3. Suppose that $t \neq 0$ and $u \neq 0$.

Each of $s$ and $v$ is nonzero because $s v=t u \neq 0$. Furthermore $s+v=1$ implies that each of $s$ and $v$ is not 1 . Thus $E$ is a matrix in the set

$$
\begin{aligned}
& \left\{\left(\begin{array}{ll}
s & t \\
u & v
\end{array}\right) \in R \mid s+v=1, s v=t u, \text { and } s, v \notin\{0,1\}\right\} \\
& =\left\{\left(\begin{array}{cc}
s & t \\
u & 1-s
\end{array}\right) \in R \mid s(1-s)=t u \text { and } s \notin\{0,1\}\right\} .
\end{aligned}
$$


(3) Suppose that $A$ is a commutative domain. Let $Q$ be the quotient field of $A$ and consider $\operatorname{Mat}_{2}(Q)$. It is well-known that if $M^{k}=0$ for some $k \geq 1$ then $M^{2}=0$, where $M \in \operatorname{Mat}_{2}(Q)$. So if $M=\left(\begin{array}{ll}s & t \\ u & v\end{array}\right) \in N(R) \backslash\{0\}$ then $M^{2}=0$. Since $M^{2}=0, M$ satisfies the equation $x^{2}=0$. But $M \neq 0$ and so $x^{2}$ is the minimal polynomial of $M$. Hence $x^{2}$ is equal to the characteristic polynomial of $M$ by the Cayley-Hamilton theorem, entailing $\operatorname{tr}(M)=0$ and $\operatorname{det}(M)=0$. Hence $s+v=0$ and $s v=t u$. This yields $-s^{2}=s(-s)=t u$.

Suppose $t u \neq 0$ (i.e., $t \neq 0$ and $u \neq 0$ ). Then $s \neq 0$ because $-s^{2}=t u$. So $M$ is of the form $\left(\begin{array}{cc}s & t \\ u & -s\end{array}\right)$ with $s \neq 0$ and $s^{2}=-t u$.

Suppose $t u=0$. Then $t=0$ or $u=0$; and $s^{2}=-t u=0$ implies $s=0$ because $A$ is a domain. Thus $M$ is one of the forms $\left(\begin{array}{ll}0 & t \\ 0 & 0\end{array}\right)$ and $\left(\begin{array}{ll}0 & 0 \\ u & 0\end{array}\right)$, where $t, u \neq 0$.

(4) and (5) are immediate consequences of (2) and (3), respectively, because $A$ is a field.

From Lemma 2.3, we can obtain the following right IQNN ring that is non-Abelian.

Theorem 2.4. If $A$ is a field then $\operatorname{Mat}_{2}(A)$ is an IQNN ring.

Proof. Let $R=\operatorname{Mat}_{2}(A)$ and suppose that $A$ is a field. Then, by Lemma 2.3(3,4),

$$
I(R)^{\prime}=\left\{\left(\begin{array}{ll}
1 & 0 \\
0 & 0
\end{array}\right),\left(\begin{array}{ll}
0 & 0 \\
0 & 1
\end{array}\right),\left(\begin{array}{ll}
1 & t \\
0 & 0
\end{array}\right),\left(\begin{array}{ll}
1 & 0 \\
u & 0
\end{array}\right),\left(\begin{array}{ll}
0 & t \\
0 & 1
\end{array}\right),\left(\begin{array}{ll}
0 & 0 \\
u & 1
\end{array}\right),\left(\begin{array}{cc}
s & t \\
t^{-1} s(1-s) & 1-s
\end{array}\right)\right\},
$$

where $s \notin\{0,1\}, t \neq 0, u \neq 0$; and

$$
N(R)=\left\{\left(\begin{array}{ll}
0 & 0 \\
0 & 0
\end{array}\right),\left(\begin{array}{ll}
0 & t \\
0 & 0
\end{array}\right),\left(\begin{array}{ll}
0 & 0 \\
u & 0
\end{array}\right),\left(\begin{array}{cc}
s & t \\
-t^{-1} s^{2} & -s
\end{array}\right) \mid s \neq 0, t \neq 0, u \neq 0\right\} .
$$

Note that the rows of $\left(\begin{array}{cc}s & t \\ t^{-1} s(1-s) & 1-s\end{array}\right)$ (resp., $\left(\begin{array}{cc}s & t \\ -t^{-1} s^{2} & -s\end{array}\right)$ ) are linearly dependent because $\left(t^{-1} s(1-s), 1-s\right)=t^{-1}(1-s)(s, t)$ (resp., $\left.\left(-t^{-1} s^{2},-s\right)=-t^{-1} s(s, t)\right)$, and that the columns of those matrices are also linearly dependent similarly.

Furthermore, for $\left(\begin{array}{cc}s & t \\ {\left[t^{-1}(1-s)\right] s} & {\left[t^{-1}(1-s)\right] t}\end{array}\right) \in I(R)^{\prime}$ and $\left(\begin{array}{cc}a & b \\ {\left[-b^{-1} a\right] a} & {\left[-b^{-1} a\right] b}\end{array}\right) \in$ $N(R)$, we have

$$
\begin{aligned}
\left(\begin{array}{cc}
s & t \\
{\left[t^{-1}(1-s)\right] s} & {\left[t^{-1}(1-s)\right] t}
\end{array}\right) & \left(\begin{array}{cc}
a & b \\
{\left[-b^{-1} a\right] a} & {\left[-b^{-1} a\right] b}
\end{array}\right) \\
& =\left(\begin{array}{cc}
b^{-1} a[s b+(-t a)] & {[s b+(-t a)]} \\
t^{-1}(1-s) b^{-1} a[s b+(-t a)] & t^{-1}(1-s)[s b+(-t a)]
\end{array}\right)=C
\end{aligned}
$$

and

$$
\begin{aligned}
\left(\begin{array}{cc}
a & b \\
{\left[-b^{-1} a\right] a} & {\left[-b^{-1} a\right] b}
\end{array}\right) & \left(\begin{array}{cc}
s & t \\
{\left[t^{-1}(1-s)\right] s} & {\left[t^{-1}(1-s)\right] t}
\end{array}\right) \\
& =\left(\begin{array}{cc}
t^{-1} s[a t+b(1-s)] & {[a t+b(1-s)]} \\
\left(-b^{-1} a\right) t^{-1} s[a t+b(1-s)] & \left(-b^{-1} a\right)[a t+b(1-s)]
\end{array}\right)=D
\end{aligned}
$$

If $s b+(-t a) \neq 0$ (resp., at $+b(1-s) \neq 0$ ) then every entry of $C$ (resp., $D$ ) is nonzero because $s \notin\{0,1\}$ and $s, t, a, b \in A \backslash\{0\}$. Suppose that $s b+(-t a) \neq 0$ and $a t+b(1-s) \neq 0$. Let $v=s b+(-t a)$ and $w=a t+b(1-s)$. Then $C=\left(\begin{array}{cc}b^{-1} a v & v \\ t^{-1}(1-s) b^{-1} a v & t^{-1}(1-s) v\end{array}\right)$ and $D=\left(\begin{array}{cc}t^{-1} s w & w \\ \left(-b^{-1} a\right) t^{-1} s w & \left(-b^{-1} a\right) w\end{array}\right)$. So we obtain

$$
C=\left(\begin{array}{cc}
-t^{-1}(1-s) v & v \\
-v^{-1}\left[t^{-1}(1-s) v\right]^{2} & t^{-1}(1-s) v
\end{array}\right)\left(\begin{array}{cc}
0 & 0 \\
b^{-1} a & 1
\end{array}\right)
$$


and

$$
D=\left(\begin{array}{cc}
1 & 0 \\
-b^{-1} a & 0
\end{array}\right)\left(\begin{array}{cc}
t^{-1} s w & w \\
-w^{-1}\left[t^{-1} s w\right]^{2} & -t^{-1} s w
\end{array}\right),
$$

noting that $\left(\begin{array}{cc}-t^{-1}(1-s) v & v \\ -v^{-1}\left[t^{-1}(1-s) v\right]^{2} & t^{-1}(1-s) v\end{array}\right),\left(\begin{array}{cc}t^{-1} s w & w \\ -w^{-1}\left[t^{-1} s w\right]^{2} & -t^{-1} s w\end{array}\right)$ are in $N(R)$ and $\left(\begin{array}{cc}0 & 0 \\ b^{-1} a & 1\end{array}\right),\left(\begin{array}{cc}1 & 0 \\ -b^{-1} a & 0\end{array}\right)$ are in $I(R)^{\prime}$. The computations for the other cases of $E N$ and $N E$ are similar or simple.

Therefore both $\left\{E N \mid E \in I(R)^{\prime}, N \in N(R)\right\}$ and $\left\{N^{\prime} E^{\prime} \mid E^{\prime} \in I(R)^{\prime}, N^{\prime} \in N(R)\right\}$ are equal to the set

$$
\left\{\left(\begin{array}{ll}
0 & 0 \\
0 & 0
\end{array}\right),\left(\begin{array}{ll}
0 & t \\
0 & 0
\end{array}\right),\left(\begin{array}{ll}
s & t \\
0 & 0
\end{array}\right),\left(\begin{array}{ll}
0 & 0 \\
0 & u
\end{array}\right),\left(\begin{array}{ll}
0 & 0 \\
s & t
\end{array}\right),\left(\begin{array}{ll}
u & 0 \\
0 & 0
\end{array}\right),\left(\begin{array}{ll}
0 & 0 \\
0 & t
\end{array}\right),\left(\begin{array}{cc}
v & 0 \\
w & 0
\end{array}\right),\left(\begin{array}{ll}
0 & v \\
0 & w
\end{array}\right),\left(\begin{array}{cc}
s & t \\
\alpha s & \alpha t
\end{array}\right)\right\},
$$

where $s, t, u, v, w, \alpha \in A \backslash\{0\}$. This proves that $R$ is both right and left IQNN.

We see another kind of right IQNN ring that is non-Abelian.

Example 2.5. (1) Let $K=\mathbb{Z}_{2}$ and $A=K\langle a, b\rangle$ be the free algebra generated by the noncommuting indeterminates $a, b$ over $K$. Let $I$ be the ideal of $A$ generated by $a^{2}-a$ and $a b-b^{2} a$. Set $R=A / I$ and identify $a$ and $b$ with their images in $R$ for simplicity. Then $a^{2}=a$ and $a b=b^{2} a$. So $R$ is non-Abelian as can be seen by $a b=b^{2} a \neq b a$.

Let $n \geq 1$. We first get $b^{2} a=a b=a^{2} b=a(a b)=a\left(b^{2} a\right)=(a b) b a=\left(b^{2} a\right) b a=b^{2}(a b) a=$ $b^{2}\left(b^{2} a\right) a=b^{4} a$; hence $a b=b^{4} a=b^{2}\left(b^{2} a\right)=b^{2}\left(b^{4} a\right)=b^{2 \cdot 3} a=b^{4}\left(b^{2} a\right)=b^{4}\left(b^{4} a\right)=b^{2 \cdot 4} a=$ $\cdots=b^{2 n} a$. This yields that for every $m \geq 5$ (say $m=4 n+l$ with $n \geq 1$ and $l=0,1,2,3$ ),

$$
b^{m} a=b^{4 n+l} a=b^{l}\left(b^{4 n} a\right)=b^{l}\left(b^{2} a\right)=b^{l+2} a \in\left\{b^{2} a, b^{3} a\right\}
$$

and

$$
a b^{2}=(a b) b=\left(b^{2} a\right) b=b^{2}(a b)=b^{2}\left(b^{2} a\right)=b^{2} a .
$$

From this result, we obtain furthermore that $a b^{3}=\left(a b^{2}\right) b=\left(b^{2} a\right) b=b^{2}(a b)=b^{2}\left(b^{2} a\right)=$ $b^{2} a$, and inductively $a b^{k+1}=\left(a b^{k}\right) b=\left(b^{2} a\right) b=b^{2}(a b)=b^{2}\left(b^{2} a\right)=b^{2} a$, where $k \geq 2$. Consequently we have now

$$
a\left(c_{1} b+c_{2} b^{2}+\cdots+c_{m} b^{m}\right)=\left(\sum_{j=1}^{m} c_{j}\right) b^{2} a \text { for all } c_{1} x+c_{2} x^{2}+\cdots+c_{m} x^{m} \in x K[x] .
$$

Therefore every $r \in R$ can be expressed by

$$
r=k_{0}+k_{1} a+\left(\alpha_{1} b a+\alpha_{2} b^{2} a+\alpha_{3} b^{3} a\right)+g(b)=k_{0}+k_{1} a+\left(\alpha_{1} b+\alpha_{2} b^{2}+\alpha_{3} b^{3}\right) a+g(b),
$$

where $k_{i}, \alpha_{j} \in K$ and $g(x) \in x K[x]$.

Let $r \in R$ with

$r=k_{0}+k_{1} a+f(b) a+g(b)$ where $f(x)=\alpha_{1} x+\alpha_{2} x^{2}+\alpha_{3} x^{3}, g(x) \in x K[x]$ and $k_{i}, \alpha_{j} \in K$.

Suppose that $r^{2}=r$, i.e., $r \in I(R)$. Then

$$
r^{2}=\left(k_{0}+k_{1} a+f(b) a+g(b)\right)^{2}=k_{0}+k_{1} a+f(b) a+g(b)=r .
$$

This entails that $k_{0}^{2}=k_{0}$ (i.e., $k_{0}=0$ or $k_{0}=1$ ) and $g(b)=g(b)^{2}$, and so $g(b)=0$ follows. Thus $r=k_{0}+k_{1} a+\left(\alpha_{1} b+\alpha_{2} b^{2}+\alpha_{3} b^{3}\right) a$.

Case I. Let $k_{0}=0$ : Then we have

$$
\begin{aligned}
r^{2} & =\left(k_{1} a+f(b) a\right)^{2}=k_{1}^{2} a+k_{1} a f(b) a+k_{1} f(b) a+f(b) a f(b) a \\
& =k_{1}^{2} a+k_{1} c b^{2} a+k_{1} f(b) a+f(b) c b^{2} a \\
& =k_{1}^{2} a+\left(k_{1} c b^{2}+k_{1} f(b)+c f(b) b^{2}\right) a=k_{1} a+f(b) a=r,
\end{aligned}
$$

where $c=\sum_{j=1}^{3} \alpha_{j}$. We also have that $k_{1}=0$ or $k_{1}=1$. 
Let $k_{1}=0$. Then $r=f(b) a$ and

$$
r^{2}=(f(b) a)^{2}=\left(c f(b) b^{2}\right) a=f(b) a=r .
$$

Here if $f(b) a \neq 0$ then $c=1$ and

$$
\left(\alpha_{1} b+\alpha_{2} b^{2}+\alpha_{3} b^{3}\right) a=\left(\alpha_{1} b+\alpha_{2} b^{2}+\alpha_{3} b^{3}\right) b^{2} a=\alpha_{1} b^{3} a+\alpha_{2} b^{2} a+\alpha_{3} b^{3} a,
$$

entailing $\alpha_{1}=0$ and $r=\left(\alpha_{2} b^{2}+\alpha_{3} b^{3}\right) a$. But $c=1$, and so $r=b^{2} a$ or $r=b^{3} a$.

Let $k_{1}=1$. Then $r=a+f(b) a$ and

$$
r^{2}=a+\left(c b^{2}+f(b)+c f(b) b^{2}\right) a=a+f(b) a=r,
$$

entailing $c b^{2} a+c f(b) b^{2} a=0$. Here if $f(b) a \neq 0$ then $c=1$ and so $f(b) a=b^{2} a$ by the same argument as the above. So $r=a+b^{2} a$ follows.

Case II. Let $k_{0}=1$ : Then we have

$$
\begin{aligned}
r^{2} & =\left(1+k_{1} a+f(b) a\right)^{2}=1+k_{1}^{2} a+k_{1} f(b) a+k_{1} a f(b) a+f(b) a f(b) a \\
& =1+k_{1}^{2} a+\left(k_{1} f(b)+k_{1} c b^{2}+c f(b) b^{2}\right) a \\
& =1+k_{1} a+f(b) a=r,
\end{aligned}
$$

entailing $k_{1}^{2}=k_{2}$ (i.e., $k_{1}=0$ or $k_{1}=1$ ) and $k_{1} f(b) a+k_{1} c b^{2} a+c f(b) b^{2} a=f(b) a$. Here assume $f(b) a \neq 0$.

Let $k_{1}=0$. Then $r=1+f(b) a$ and $c f(b) b^{2} a=f(b) a$. Hence $c=1$ and so $r=1+b^{2} a$ or $r=1+b^{3} a$ by the similar computation to the above.

Let $k_{1}=1$. Then $r=1+a+f(b) a$ and $f(b) a+c b^{2} a+c f(b) b^{2} a=f(b) a$, entailing $c=0$ or $b^{2} a=f(b) b^{2} a$ (when $c=1$ ). So if $c=1$ then $f(b) a=b^{2} a$.

Consequently we now have

$$
I(R)=\left\{0,1, a, b^{2} a, b^{3} a, a+b^{2} a, 1+a, 1+b^{2} a, 1+b^{3} a, 1+a+b^{2} a\right\} .
$$

Next we examine the set of nilpotents in $R$. Suppose $r^{n}=0$ for $r=k_{0}+k_{1} a+f(b) a+g(b)$ where $f(x)=\alpha_{1} x+\alpha_{2} x^{2}+\alpha_{3} x^{3}, g(x) \in x K[x]$ and $k_{i}, \alpha_{j} \in K$. Then $g(b)=0$ because $r^{n}=k_{0}+k_{1} a+\left(\gamma_{1} b+\gamma_{2} b^{2}+\gamma_{3} b^{3}\right) a+g(b)^{n}=0$ with $\gamma_{j} \in K$. Similarly, we get $k_{0}=0$ and $k_{1}=0$. Thus $r=f(b) a$, and by the fact that $a f(b)=c b^{2} a$, we have

$$
r^{n}=f(b) c^{n-1} b^{2} a=c^{n-1} \alpha_{2} b^{2} a+\alpha^{n-1}\left(\alpha_{1}+\alpha_{3}\right) b^{3} a=c \alpha_{2} b^{2} a+c\left(\alpha_{1}+\alpha_{3}\right) b^{3} a=0,
$$

where $c=\sum_{j=1}^{3} \alpha_{j}$. It then follows that $c \alpha_{2}=0$ and $c\left(\alpha_{1}+\alpha_{3}\right)=0$. If $c=0$, then we are done. Assume $c \neq 0$. Then $\alpha_{2}=0$ and $\alpha_{1}+\alpha_{3}=0$. This yields $c=0$, a contradiction. Consequently, we must have $c=0$ for $r^{n}$ to be zero. Moreover if $c=0$ then $r^{2}=0$. Therefore

$$
\begin{aligned}
N(R) & =\left\{\alpha_{1} b a+\alpha_{2} b^{2} a+\alpha_{3} b^{3} a \mid \alpha_{1}, \alpha_{2}, \alpha_{3} \in K \text { with } \alpha_{1}+\alpha_{2}+\alpha_{3}=0\right\} \\
& =\left\{0, b a+b^{2} a, b a+b^{3} a, b^{2} a+b^{3} a\right\} .
\end{aligned}
$$

Now we claim that $R$ is an IQNN ring. Let $e \in I(R)^{\prime}$ and $n=\alpha_{1} b a+\alpha_{2} b^{2} a+\alpha_{3} b^{3} a \in N(R)$. Then $\alpha_{1}+\alpha_{2}+\alpha_{3}=0$.

If $e \in\left\{a, b^{2} a, b^{3} a, a+b^{2} a\right\}$ then $e n=0$ because $a n=0$, and so $e n=0 \in N(R) f$ for any $f \in I(R)^{\prime}$.

Next let $e \in\left\{1+a, 1+b^{2} a, 1+b^{3} a, 1+a+b^{2} a\right\}$. Then

$(1+a) n=n=n a ;\left(1+b^{2} a\right) n=n=n a ;\left(1+b^{3} a\right) n=n=n a ;$ and $\left(1+a+b^{2} a\right) n=n=n a$.

Therefore $R$ is right IQNN. 
Let $e \in\left\{a, b^{2} a, b^{3} a, a+b^{2} a\right\}$. Then

$$
\begin{aligned}
n a & =n=(1+a) n ; \\
n b^{2} a & \left.=\alpha_{2} b^{2} a+\left(\alpha_{1}+\alpha_{3}\right) b^{3} a=\alpha_{2} b^{2} a+\alpha_{2} b^{3} a\right)=(1+a)\left(\alpha_{2} b^{2} a+\alpha_{2} b^{3} a\right) ; \\
n b^{3} a & \left.=\alpha_{2} b^{2} a+\left(\alpha_{1}+\alpha_{3}\right) b^{3} a=\alpha_{2} b^{2} a+\alpha_{2} b^{3} a\right)=(1+a)\left(\alpha_{2} b^{2} a+\alpha_{2} b^{3} a\right) ; \text { and } \\
n\left(a+b^{2} a\right) & \left.=\alpha_{1} b a+\alpha_{1} b^{3} a\right)=(1+a)\left(\alpha_{1} b a+\alpha_{1} b^{3} a\right),
\end{aligned}
$$

noting that $\left.\alpha_{2} b^{2} a+\alpha_{2} b^{3} a\right), \alpha_{1} b a+\alpha_{1} b^{3} a \in N(R)$ because $c=\alpha_{1}+\alpha_{2}+\alpha_{3}=0$.

Next let $e \in\left\{1+a, 1+b^{2} a, 1+b^{3} a, 1+a+b^{2} a\right\}$. Then

$$
\begin{aligned}
n(1+a) & =0 \in f N(R) \text { for any } f \in I(R)^{\prime} ; \\
n\left(1+b^{2} a\right) & =\alpha_{1} b a+\alpha_{1} b^{3} a=(1+a)\left(\alpha_{1} b a+\alpha_{1} b^{3} a\right) ; \\
n\left(1+b^{3} a\right) & =\alpha_{1} b a+\alpha_{1} b^{3} a=(1+a)\left(\alpha_{1} b a+\alpha_{1} b^{3} a\right) ; \text { and } \\
n\left(1+a+b^{2} a\right) & =n b^{2} a=(1+a)\left(\alpha_{2} b^{2} a+\alpha_{2} b^{3} a\right),
\end{aligned}
$$

by a similar method to the above. Therefore $R$ is left IQNN.

McCoy [18] called a ring $R \pi$-regular if for each $a \in R$ there exist a positive integer $n$, depending on $a$, and $b \in R$ such that $a^{n}=a^{n} b a^{n}$. Note that $\operatorname{Mat}_{n}(D)$ over a division ring $D$ for $n \geq 2$ is $\pi$-regular by [8, Theorem 1.7]. If a ring $R$ is Abelian $\pi$-regular, then $N(R)=N^{*}(R)=J(R)$ by [19, Theorem 2]. However this result is not valid for right IQNN $\pi$-regular rings. Consider the ring $R=\operatorname{Mat}_{2}(A)$ over a field $A$ in Theorem 2.4. Then $R$ is IQNN $\pi$-regular, but $0=N^{*}(R) \subsetneq N(R)$ and $J(R)=0$.

The one-sided IQNN property is not left-right symmetric by the following.

Example 2.6. We apply the construction and argument in [14, Example 2.3(1,2)]. Let $K=\mathbb{Z}_{2}$ and $A=K\langle a, b\rangle$ be the free algebra with noncommuting indeterminates $a, b$ over $K$.

(1) Consider the ideal $I$ of $A$ generated by $b a, a^{2}-a, b^{2}$ and let $R_{1}=A / I$ and identify the elements in $A$ with their images in $R_{1}$ for simplicity. Then $a^{2}=a$ and $b a=0=b^{2}$ in $R_{1}$. By help of [14, Example 2.3(1)], every element $r \in R$ is of the form $r=\alpha_{0}+\alpha_{1} a+\alpha_{2} a b+\alpha_{3} b$, where $\alpha_{0}, \alpha_{1}, \alpha_{2}, \alpha_{3} \in K$.

Let $(1+f)^{2}=1+f$ with $f=\alpha_{1} a+\alpha_{2} a b+\alpha_{3} b$. Then $1+2 f+f^{2}=1+f^{2}=1+f$ and $f^{2}=f$ follows. Thus

$$
I\left(R_{1}\right)^{\prime}=\{\alpha+a+\gamma a b \mid \alpha, \gamma \in K\}=\left\{1+a+\gamma a b, a+\gamma^{\prime} a b \mid \gamma, \gamma^{\prime} \in K\right\}
$$

by help of the computation [14, Example 2.3(1)]. Moreover, $N\left(R_{1}\right)=\{\alpha a b+\beta b \mid \alpha, \beta \in K\}$ by the computation to [14, Example 2.3(1)]. In fact, $N\left(R_{1}\right)$ is an ideal of $R$. We directly obtain that

$$
S_{1}=\left\{e n \mid e \in I\left(R_{1}\right)^{\prime}, n \in N\left(R_{1}\right)\right\}=\{\delta b+\delta a b, \eta a b \mid \delta, \eta \in K\}
$$

and

$$
S_{2}=\left\{n^{\prime} e^{\prime} \mid n^{\prime} \in N\left(R_{1}\right), e^{\prime} \in I\left(R_{1}\right)^{\prime}\right\}=N\left(R_{1}\right) .
$$

Thus $S_{1} \subsetneq S_{2}$, and this implies that $R_{1}$ is right IQNN but not left IQNN.

(2) Consider the ideal $J$ of $A$ generated by $a b, a^{2}-a, b^{2}$. Let $R_{2}=B / J$ and identify the elements in $A$ with their images in $R_{2}$ for simplicity. Then $R_{2}$ is left IQNN but not right IQNN by a similar argument to (1).

As a generalization of reduced rings, Marks [17] called a ring $R N I$ if $N^{*}(R)=N(R)$. Note that a ring $R$ is NI if and only if $N(R)$ forms an ideal if and only if $R / N^{*}(R)$ is reduced.

The class of NI rings and the class of right IQNN rings do not imply each other by next example. 
Example 2.7. (1) The IQNN ring $M_{a t}(A)$ over a field $A$ in Theorem 2.4 is not NI clearly.

(2) Let $S$ be a domain and consider a ring $R=D_{2}(S)$. Then $R$ is NI since $N^{*}(R)=$ $\left(\begin{array}{cc}0 & D \\ 0 & 0\end{array}\right)=N(R)$. Thus $T_{n}(R)$ for all $n \geq 2$ is NI by [11, Proposition 4.1(1)], but it is neither left nor right IQNN by Example 3.2 to follow.

Example 2.6 illuminates that the IQNN property is not left-right symmetric, even if given rings are NI. Indeed, each $N\left(R_{i}\right)$ forms an ideal of $R_{i}$ for $i=1,2$.

Let $R$ be a right IQNN ring and suppose that $e \in I(R)^{\prime}$ and $a \in N(R)$. Then $e a=b f$ for some $b \in N(R)$ and $f \in I(R)^{\prime}$. Here $e a=b f=(b f) f=(e a) f$. We will freely us this fact in the procedure. Moreover, we note that if a ring $R$ is NI then $e a, a e \in N(R)$ for all $e \in I(R)$ and $a \in N(R)$. So we get the following.

Proposition 2.8. Let $R$ be an NI ring. Then the following conditions are equivalent:

(1) $R$ is right IQNN;

(2) for any $e \in I(R)^{\prime}$ and $a \in N(R)$, there exists $f \in I(R)^{\prime}$ such that ea $\in N(R) f$;

(3) for any $e \in I(R)^{\prime}$ and $a \in N(R)$, there exists $f \in I(R)^{\prime}$ such that ea $=$ eaf.

We see in the following a condition under which the IQNN property is left-right symmetric. Recall that an involution on a ring $R$ is a function $*: R \rightarrow R$ which satisfies the properties that $(x+y)^{*}=x^{*}+y^{*},(x y)^{*}=y^{*} x^{*}, 1^{*}=1$, and $\left(x^{*}\right)^{*}=x$ for all $x, y \in R$. We get $0^{*}=0$ because $0^{*}=(0+0)^{*}=0^{*}+0^{*}$, and $e^{*}=(e e)^{*}=e^{*} e^{*}$ implies that $e^{*} \in I(R)$ for all $e \in I(R)$.

Proposition 2.9. Let $R$ be a ring with an involution *. Then the following conditions are equivalent:

(1) $R$ is right IQNN;

(2) $R$ is left IQNN.

Proof. First note that $e^{*} \in I(R)^{\prime}$ for all $e \in I(R)^{\prime}$. Note that $e \neq 0$ and $e \neq 1$, entailing $1-e \in I(R)^{\prime}$. If $e^{*}=0$ then $e=\left(e^{*}\right)^{*}=0^{*}=0$, a contradiction. So $e^{*} \neq 0$. If $e^{*}=1$ then $0=\left(0^{*}\right)^{*}=\left((e(1-e))^{*}\right)^{*}=\left((1-e)^{*} e^{*}\right)^{*}=\left((1-e)^{*}\right)^{*}=1-e$, a contradiction. So $e^{*} \neq 1$.

Next, if $a \in N(R)$, then $a^{k}=0$ for $k \geq 1$. Since $\left(a^{k}\right)^{*}=\left(a^{*}\right)^{k}$ and $0^{*}=0, a^{*} \in N(R)$.

$(1) \Leftrightarrow(2)$ : Let $R$ be a right IQNN. For $a \in N(R), e \in I(R)^{\prime}$, we have $a^{*} \in N(R)$, $e^{*} \in I(R)^{\prime}$. So there exist $b \in N(R)$ and $f \in I(R)^{\prime}$ such that $e^{*} a^{*}=b f$. Thus we have

$$
a e=\left((a e)^{*}\right)^{*}=\left(e^{*} a^{*}\right)^{*}=(b f)^{*}=f^{*} b^{*} .
$$

But $f^{*} \in I(R)^{\prime}$, so $R$ is left IQNN. The converse can be similarly proved.

Following [5], a ring $R$ is called right (resp., left) quasi-Abelian provided that either $I(R)^{\prime}$ is empty, or else for any $(e, a) \in I(R)^{\prime} \times R$ (resp., $\left.(a, e) \in R \times I(R)^{\prime}\right)$ there exists $(b, f) \in R \times I(R)^{\prime}$ (resp., $\left.(f, b) \in I(R)^{\prime} \times R\right)$ such that $e a=b f$ (resp., $\left.a e=f b\right)$.

The concept of right IQNN coincides with one of right quasi-Abelian when $R$ is restricted to $N(R)$. But there exists a right IQNN ring which is not right quasi-Abelian, even though it is NI, as we see in the following.

Example 2.10. Consider $R=\left(\begin{array}{cc}\mathbb{Z} & \mathbb{Z}_{2} \\ 0 & \mathbb{Z}\end{array}\right)$. Then $R$ is not right quasi-Abelian by [5, Example 1.8]. Note that

$$
I(R)^{\prime}=\left\{\left(\begin{array}{ll}
0 & b \\
0 & 1
\end{array}\right),\left(\begin{array}{ll}
1 & b^{\prime} \\
0 & 0
\end{array}\right) \mid b, b^{\prime} \in \mathbb{Z}_{2}\right\} \text { and } N(R)=\left(\begin{array}{cc}
0 & \mathbb{Z}_{2} \\
0 & 0
\end{array}\right) .
$$

Then $\left\{A B \mid A \in I(R)^{\prime}, B \in N(R)\right\}=N(R)=\left\{C D \mid C \in N(R), D \in I(R)^{\prime}\right\}$, and so $R$ is both left and right IQNN. Moreover, $R$ is NI since $N_{*}(R)=N^{*}(R)=N(R)$. 

$R_{\gamma}$.

Use $\prod_{\gamma \in \Gamma} R_{\gamma}$ (resp., $\bigoplus_{\gamma \in \Gamma} R_{\gamma}$ ) to denote the direct product (resp., direct sum) of rings

Proposition 2.11. (1) Let $\left\{R_{\gamma} \mid \gamma \in \Gamma\right\}$ be a family of rings, and $R$ be the subring of $\prod_{\gamma \in \Gamma} R_{\gamma}$ generated by $\bigoplus_{\gamma \in \Gamma} R_{\gamma}$ and ${ }^{1} \prod_{\gamma \in \Gamma} R_{\gamma}$. If $R_{\gamma}$ is right IQNN for all $\gamma \in \Gamma$ then $R$ is right IQNN.

(2) Let $R$ be a ring and $e \in I(R)^{\prime}$ be central. If $R$ is right IQNN then both $e R$ and $(1-e) R$ are right IQNN.

Proof. (1) Note first $N(R)=\bigoplus_{\gamma \in \Gamma} N\left(R_{\gamma}\right)$. We refer to the argument of [5, Theorem $2.2(2)]$. Assume that $R_{\gamma}$ is right IQNN for all $\gamma$. Consider $\left(e_{\gamma}\right)_{\gamma \in \Gamma} \in I(R)^{\prime}$ and $\left(r_{\gamma}\right)_{\gamma \in \Gamma} \in$ $N(R)$. Then we have the following two cases:

Case I. There exists a finite nonempty subset $\Gamma_{1}$ of $\Gamma$ such that $e_{\alpha} \in I\left(R_{\alpha}\right)^{\prime}$ for all $\alpha \in \Gamma_{1}$ and $e_{\beta} \in\left\{0_{R_{\beta}}, 1_{R_{\beta}}\right\}$ for all $\beta \in \Gamma \backslash \Gamma_{1}$.

Since every $R_{\gamma}$ is right IQNN, there exists $g_{\alpha} \in I\left(R_{\alpha}\right)^{\prime}$ and $t_{\alpha} \in N\left(R_{\alpha}\right)$ such that $e_{\alpha} r_{\alpha}=t_{\alpha} g_{\alpha}$, for all $\alpha \in \Gamma_{1}$. Let $\left(f_{\gamma}\right)_{\gamma \in \Gamma} \in I(R)$ and $\left(s_{\gamma}\right)_{\gamma \in \Gamma} \in N(R)$ be such that

$$
f_{\alpha}=g_{\alpha} \text { for all } \alpha \in \Gamma_{1} \text { and } f_{\beta}=e_{\beta} \text { for all } \beta \in \Gamma \backslash \Gamma_{1} \text {; }
$$

and

$$
s_{\alpha}=t_{\alpha} \text { for all } \alpha \in \Gamma_{1} \text { and } s_{\beta}=r_{\beta} \text { for all } \beta \in \Gamma \backslash \Gamma_{1} .
$$

Then $\left(e_{\gamma}\right)_{\gamma \in \Gamma}\left(r_{\gamma}\right)_{\gamma \in \Gamma}=\left(s_{\gamma}\right)_{\gamma \in \Gamma}\left(f_{\gamma}\right)_{\gamma \in \Gamma}$ because $e_{\alpha} r_{\alpha}=t_{\alpha} g_{\alpha}=s_{\alpha} f_{\alpha}$ and $e_{\beta} r_{\beta}=r_{\beta} e_{\beta}=$ $s_{\beta} f_{\beta}$. Moreover $\left(f_{\gamma}\right)_{\gamma \in \Gamma}$ and $\left(s_{\gamma}\right)_{\gamma \in \Gamma}$ are contained in $I(R)^{\prime}$ and $N(R)$, respectively.

Case II. There exists a finite nonempty subset $\Gamma_{2}$ of $\Gamma$ such that $e_{\alpha^{\prime}}=0_{R_{\alpha^{\prime}}}$ for all $\alpha^{\prime} \in \Gamma_{2}$ and $e_{\beta^{\prime}}=1_{R_{\beta^{\prime}}}$ for all $\beta^{\prime} \in \Gamma \backslash \Gamma_{2}$. Then $\left(e_{\gamma}\right)_{\gamma \in \Gamma}$ is central in $R$, and $\left(e_{\gamma}\right)_{\gamma \in \Gamma}\left(r_{\gamma}\right)_{\gamma \in \Gamma}=$ $\left(r_{\gamma}\right)_{\gamma \in \Gamma}\left(e_{\gamma}\right)_{\gamma \in \Gamma}$ follows.

Therefore $R$ is right IQNN.

(2) Notice that

$$
\begin{aligned}
N(e R) & =e N(R), I(e R) \subseteq I(R)^{\prime} \cup\{0\} ; \text { and } \\
N((1-e) R) & =(1-e) N(R), I((1-e) R) \subseteq I(R)^{\prime} \cup\{0\} .
\end{aligned}
$$

Suppose that $R$ is right IQNN, and let ef $\in I(e R)^{\prime}$ and $e s \in N(e R)$. If $(e f)(e s)=0$ then we are done. So assume $(e f)(e s) \neq 0$. Since $R$ is right IQNN, there exist $t \in N(R)$ and $h \in I(R)^{\prime}$ such that $(e f)(e s)=t h$, entailing $(e f)(e s)=(e t)(e h)$. But et $\in N(e R)$, and $e h \in I(e R)^{\prime}$ because $e h \neq 0$. Thus $e R$ is right IQNN. Similarly, we can prove that $(1-e) R$ is right IQNN.

For finite rings we obtain a useful information. Recall that if $M^{k}=0$ for some $k \geq 1$ then $M^{2}=0$, where $M \in \operatorname{Mat}_{2}(F)$ and $F$ is a field.

Corollary 2.12. Let $R$ be a finite ring of bounded index (of nilpotency) $\leq 2$. Then $R / J(R)$ is an IQNN ring.

Proof. Let $R$ be a finite ring. Then $R / J(R)$ is a finite direct product of $M_{a t} t_{n_{i}}\left(F_{i}\right)$ where $F_{i}$ is a finite field for all $i$. Here $n_{i}$ is either 1 or 2 because $R$ (hence $R / J(R)$ ) is of bounded index (of nilpotency) $\leq 2$. This completes the proof by help of Theorem 2.4 and Proposition 2.11(1).

\section{Properties and examples of right IQNN rings}

In this section, we observe the structures of several sorts of right IQNN rings which are related to matrix rings, factor rings, and polynomial rings. In the procedure, we also study the structure of NR rings (i.e., rings in which the nilpotents form a subring) with respect to the Abelian (hence IQNN) property. The work, in this section, enables us to show that the class of right IQNN rings is quite large. 
Theorem 3.1. If $R$ is a reduced ring then $T_{n}(R)$ is an IQNN ring for all $n \geq 2$.

Proof. Let $R$ be a reduced ring and $T=T_{n}(R)$ for $n \geq 2$. Then $N(T)=\left\{\left(a_{i j}\right) \in T \mid\right.$ $a_{i i}=0$ for all $\left.i=1,2, \ldots, n\right\}=N_{*}(T)=N^{*}(T)$. Let $A \in N(T)$ and $E \in I(T)^{\prime}$. Then clearly $A E, E A \in N(T), A E=\left(b_{i j}\right)$ and $E A=\left(c_{i j}\right)$ say. Next set

$$
F_{1}=E_{11}+E_{22}+\cdots+E_{(n-1)(n-1)} \text { and } F_{2}=E_{22}+E_{33}+\cdots+E_{n n} .
$$

Then $F_{1}, F_{2} \in I(T)^{\prime}$ and moreover $A E=F_{1}\left(b_{i j}\right), E A=\left(c_{i j}\right) F_{2}$. Thus $T$ is IQNN.

The condition " $R$ is a reduced ring" in Theorem 3.1 is not superfluous as we see in the following.

Example 3.2. (1) Let $S$ be a domain and consider the non-reduced ring $R=D_{2}(S)$. By [10, Lemma 2], $R$ is Abelian with $I(R)=\left\{\left(\begin{array}{ll}0 & 0 \\ 0 & 0\end{array}\right),\left(\begin{array}{ll}1 & 0 \\ 0 & 1\end{array}\right)\right\}$ and so it is IQNN. Next set $T=T_{2}(R)$. Then we have $N(R)=\left\{\left(a_{i j}\right) \in R \mid a_{11}=a_{22}=0\right\}$ and $N(T)=\left\{\left(b_{i j}\right) \in T \mid\right.$ $\left.b_{11}, b_{12} \in N(R)\right\}$. We claim that $T$ is neither right nor left IQNN.

Consider two matrices

$$
E=\left(\begin{array}{ll}
\left(\begin{array}{ll}
1 & 0 \\
0 & 1
\end{array}\right) & \left(\begin{array}{ll}
0 & 0 \\
0 & 0
\end{array}\right) \\
\left(\begin{array}{ll}
0 & 0 \\
0 & 0
\end{array}\right) & \left(\begin{array}{ll}
0 & 0 \\
0 & 0
\end{array}\right)
\end{array}\right) \text { and } A=\left(\begin{array}{ll}
\left(\begin{array}{ll}
0 & 1 \\
0 & 0
\end{array}\right) & \left(\begin{array}{ll}
1 & 1 \\
0 & 1
\end{array}\right) \\
\left(\begin{array}{ll}
0 & 0 \\
0 & 0
\end{array}\right) & \left(\begin{array}{ll}
0 & 0 \\
0 & 0
\end{array}\right)
\end{array}\right)
$$

in $T$. Then $E \in I(T)^{\prime}$ and $A \in N(T)$ with $E A=\left(\begin{array}{ll}\left(\begin{array}{ll}0 & 1 \\ 0 & 0\end{array}\right) & \left(\begin{array}{ll}1 & 1 \\ 0 & 1\end{array}\right) \\ \left(\begin{array}{ll}0 & 0 \\ 0 & 0\end{array}\right) & \left(\begin{array}{ll}0 & 0 \\ 0 & 0\end{array}\right)\end{array}\right)=A$. Assume on the contrary that there exist $F \in I(T)^{\prime}$ and $B \in N(T)$ such that $A=E A=B F$, say $B=\left(\begin{array}{cc}B_{1} & B_{2} \\ 0 & B_{3}\end{array}\right)$ and $F=\left(\begin{array}{cc}F_{1} & F_{2} \\ 0 & F_{3}\end{array}\right)$ with $B_{j}, F_{j} \in R$ for $j=1,2,3$. Then $B_{j} \in N(R)$ and $F_{j}^{2}=F_{j}$ for $j=1,3$ such that

$$
B_{1} F_{1}=\left(\begin{array}{ll}
0 & 1 \\
0 & 0
\end{array}\right) \text { and } B_{1} F_{2}+B_{2} F_{3}=\left(\begin{array}{ll}
1 & 1 \\
0 & 1
\end{array}\right) .
$$

The first equality forces

$$
B_{1}=\left(\begin{array}{ll}
0 & 1 \\
0 & 0
\end{array}\right) \text { and } F_{1}=\left(\begin{array}{ll}
1 & 0 \\
0 & 1
\end{array}\right)
$$

because $I(R)=\left\{\left(\begin{array}{ll}0 & 0 \\ 0 & 0\end{array}\right),\left(\begin{array}{ll}1 & 0 \\ 0 & 1\end{array}\right)\right\}$. This yields

$$
B_{2} F_{3}=\left(\begin{array}{ll}
1 & 1 \\
0 & 1
\end{array}\right)-B_{1} F_{2}=\left(\begin{array}{ll}
1 & 1 \\
0 & 1
\end{array}\right)-\left(\begin{array}{ll}
0 & 1 \\
0 & 0
\end{array}\right) F_{2} \notin N(T)
$$

because $\left(\begin{array}{ll}0 & 1 \\ 0 & 0\end{array}\right) F_{2} \in N(T)$. But $F_{3} \in I(R)$ and $F_{3} \neq 0$, and so $F_{3}=\left(\begin{array}{ll}1 & 0 \\ 0 & 1\end{array}\right)$. From these

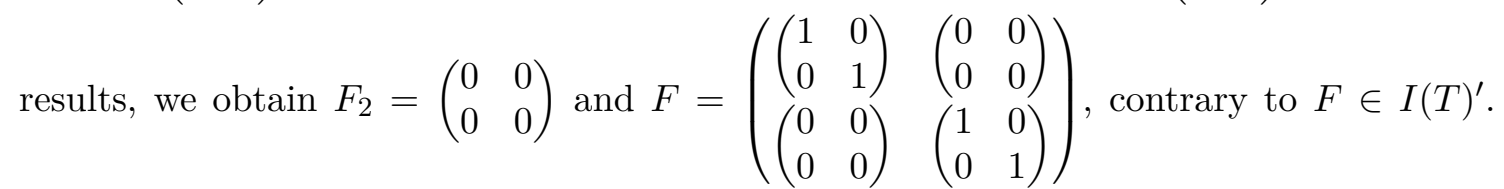
Therefore $T$ is not right IQNN. Similar proof can be done for the case of $T$ being not left IQNN.

(2) Consider a non-reduced commutative rings $R=\mathbb{Z}_{4}$. Then $R$ is clearly IQNN with $I(R)=\{0,1\}$. Consider $T=T_{2}(R)$. Then $I(T)^{\prime}=\left\{\left(\begin{array}{ll}1 & a \\ 0 & 0\end{array}\right),\left(\begin{array}{ll}0 & b \\ 0 & 1\end{array}\right) \mid a, b \in R\right\}$. For 
$E=\left(\begin{array}{ll}1 & 0 \\ 0 & 0\end{array}\right) \in I(T)^{\prime}$ and $A=\left(\begin{array}{ll}2 & 1 \\ 0 & 0\end{array}\right) \in N(T)$, we have $E A=\left(\begin{array}{ll}2 & 1 \\ 0 & 0\end{array}\right)$. However $\left(\begin{array}{ll}2 & 1 \\ 0 & 0\end{array}\right)\left(\begin{array}{ll}1 & a \\ 0 & 0\end{array}\right)=\left(\begin{array}{cc}2 & 2 a \\ 0 & 0\end{array}\right)$ and $\left(\begin{array}{ll}2 & 1 \\ 0 & 0\end{array}\right)\left(\begin{array}{cc}0 & b \\ 0 & 1\end{array}\right)=\left(\begin{array}{cc}0 & 2 b+1 \\ 0 & 0\end{array}\right)$; hence $E A \neq E A F$ for all $F \in I(T)^{\prime}$. Thus $T$ is not right IQNN by the argument before Proposition 2.8. Similarly, it can be shown that $T$ is not left IQNN.

As an application of Theorem 3.1, we assert in the following that the class of right IQNN rings is not closed under subrings.

Example 3.3. Let $S$ be a domain and $T_{0}=T_{4}(S)$. Then $R_{0}$ is IQNN by Theorem 3.1. Consider the ring $T=T_{2}(R)$ with $R=D_{2}(S)$ as in Example 3.2(1). Then $T$ is not right IQNN by the argument in Example 3.2(1). Note that $T$ is a subring of $T_{0}$.

By hint of Example 3.2, we get the following which is in part a converse of Theorem 3.1 .

Theorem 3.4. Let $R$ be a ring with $I(R)=\{0,1\}$ and $n \geq 2$. Then the following conditions are equivalent:

(1) $T_{n}(R)$ is right IQNN;

(2) $R$ is reduced;

(3) $T_{n}(R)$ is left IQNN.

Proof. $(1) \Rightarrow(2)$ : Suppose that $T=T_{n}(R)$ is right IQNN. Assume on the contrary that $a^{2}=0$ for some $0 \neq a \in R$. Consider two matrices $E=\sum_{i=1}^{n-1} E_{i i}$ and $A=$ $a\left(\sum_{i=1}^{n-1} E_{i i}\right)+E_{(n-1) n}$ in $T$. Then $E \in I(T)^{\prime}$ and $A \in N(T)$ such that $E A=A$.

Since $T$ is right IQNN, there exist $F \in I(T)^{\prime}$ and $B \in N(T)$ such that $E A=B F$, say $B=\left(b_{i j}\right)$ and $F=\left(f_{i j}\right)$. Then $b_{i i} \in N(R)$ and $f_{i i} \in I(R)$ such that $b_{i i} f_{i i}=a$ for all $i=1,2, \ldots, n-1$ and $b_{n n} f_{n n}=0$. Since $I(R)=\{0,1\}$, every $f_{i i}$ is 0 or 1 . This forces $f_{i i}=1$ (hence $b_{i i}=a$ ) for all $i=1,2, \ldots, n-1$. But $F \in I(T)^{\prime}$ and $f_{n n}=0$ follows. Since $F^{2}=F$, we can obtain directly that $f_{i j}=0$ for all $i, j \in\{1,2, \ldots, n-1\}$ with $i \neq j$; that is, $F=E_{11}+E_{22}+\cdots+E_{(n-1)(n-1)}+f_{1 n} E_{1 n}+f_{2 n} E_{2 n}+\cdots+f_{(n-1) n} E_{(n-1) n}$.

This yields $1=b_{(n-1)(n-1)} f_{(n-1) n}+b_{(n-1) n} f_{n n}=a f_{(n-1) n}$, so we get $0 \neq a=a^{2} f_{(n-1) n}=$ 0 , a contradiction. Thus $R$ is reduced. 3.1.

The proof of $(3) \Rightarrow(2)$ is similar. Both $(2) \Rightarrow(1)$ and $(2) \Rightarrow(3)$ are proved by Theorem

Considering a reduced ring $R$ with $I(R)=\{0,1\}$ as in Theorem 3.4, one may ask whether such a reduced ring is a domain. However the answer is negative by the following.

Example 3.5. Consider the polynomial ring $P=\mathbb{Z}_{6}[x]$. Then $P$ is a reduced ring, and $I(P)=\{0,1,3,4\}$ by $[13$, Lemma 8$]$. Next set

$$
R=\{0,1\}+\mathbb{Z}_{6}[x] x .
$$

Then $R$ is a reduced ring with $I(R)=\{0,1\}$, but it is not a domain.

The following example shows that the converse of Theorem 3.1 does not hold in general as well as the condition " $R$ is a ring with $I(R)=\{0,1\}$ " in Theorem 3.4 is not superfluous.

Example 3.6. Let $R_{1}$ be the right IQNN ring in Example 2.6(1) with

$$
N\left(R_{1}\right)=\{\alpha a b+\beta b \mid \alpha, \beta \in K\}
$$

and

$$
I\left(R_{1}\right)=\left\{0,1,1+a+\gamma a b, a+\gamma^{\prime} a b \mid \alpha, \gamma, \gamma^{\prime} \in K\right\} .
$$

We will use the argument in Example 2.6(1) without referring. Then $R_{1}$ is clearly not reduced. 
Set $T=T_{2}\left(R_{1}\right)$. Then

$$
N(T)=\left\{\left(\begin{array}{ll}
c & s \\
0 & d
\end{array}\right) \mid c, d \in N\left(R_{1}\right) \text { and } s \in R_{1}\right\},
$$

and every idempotent $E$ in $I(T)^{\prime}$ is contained in the set

$$
\left\{\left(\begin{array}{cc}
e & r \\
0 & f^{\prime}
\end{array}\right),\left(\begin{array}{cc}
e^{\prime} & r \\
0 & f
\end{array}\right),\left(\begin{array}{cc}
1 & r \\
0 & f^{\prime}
\end{array}\right),\left(\begin{array}{cc}
e^{\prime} & r \\
0 & 1
\end{array}\right)\right\},
$$

where $e, f \in I\left(R_{1}\right)^{\prime}, e^{\prime}, f^{\prime} \in\{0\} \cup I\left(R_{1}\right)^{\prime}$, and $r \in R_{1}$. Note that $N\left(R_{1}\right)=N^{*}\left(R_{1}\right)$ and $\left\{n^{\prime} e^{\prime} \mid n^{\prime} \in N\left(R_{1}\right), e^{\prime} \in I\left(R_{1}\right)^{\prime}\right\}=N\left(R_{1}\right)$.

We will show that $T$ is a right IQNN ring. Let $E \in I(T)^{\prime}$ and $A=\left(\begin{array}{cc}c & s \\ 0 & d\end{array}\right) \in N(T)$. Note $c, d \in N\left(R_{1}\right)$ and $N\left(R_{1}\right)=N^{*}\left(R_{1}\right)$.

(i) Consider the case of $E=\left(\begin{array}{cc}e & r \\ 0 & f^{\prime}\end{array}\right)$. Then $E A=\left(\begin{array}{cc}e c & e s+r d \\ 0 & f^{\prime} d\end{array}\right)$. Since $R_{1}$ is right IQNN, $e c=c_{1} e_{1}$ for some $c_{1} \in N\left(R_{1}\right)$ and $e_{1} \in I\left(R_{1}\right)^{\prime}$. So $E A=\left(\begin{array}{cc}c_{1} & e s+r d \\ 0 & f^{\prime} d\end{array}\right)\left(\begin{array}{cc}e_{1} & 0 \\ 0 & 1\end{array}\right)$, noting that $\left(\begin{array}{cc}c_{1} & e s+r d \\ 0 & f^{\prime} d\end{array}\right) \in N(T)$ and $\left(\begin{array}{cc}e_{1} & 0 \\ 0 & 1\end{array}\right) \in I(T)^{\prime}$.

(ii) Consider the case of $E=\left(\begin{array}{ll}e^{\prime} & r \\ 0 & f\end{array}\right)$. Then $E A=\left(\begin{array}{cc}e^{\prime} c & e^{\prime} s+r d \\ 0 & f d\end{array}\right)$. If $e^{\prime}=0$ then $E A=\left(\begin{array}{ll}0 & r d \\ 0 & f d\end{array}\right)=\left(\begin{array}{ll}0 & r d \\ 0 & f d\end{array}\right)\left(\begin{array}{ll}0 & 0 \\ 0 & 1\end{array}\right)$, noting that $\left(\begin{array}{ll}0 & r d \\ 0 & f d\end{array}\right) \in N(T)$ and $\left(\begin{array}{ll}0 & 0 \\ 0 & 1\end{array}\right) \in I(T)^{\prime}$. Suppose $e^{\prime} \neq 0$. Since $R_{1}$ is right IQNN, $e^{\prime} c=c_{2} e_{2}$ for some $c_{2} \in N\left(R_{1}\right)$ and $e_{2} \in$ $\{0\} \cup I\left(R_{1}\right)^{\prime}$. So $E A=\left(\begin{array}{cc}c_{2} & e s+r d \\ 0 & f d\end{array}\right)\left(\begin{array}{cc}e_{2} & 0 \\ 0 & 1\end{array}\right)$, noting that $\left(\begin{array}{cc}c_{1} & e^{\prime} s+r d \\ 0 & f d\end{array}\right) \in N(T)$ and $\left(\begin{array}{cc}e_{2} & 0 \\ 0 & 1\end{array}\right) \in I(T)^{\prime}$

(iii) Consider the case of $E=\left(\begin{array}{cc}1 & r \\ 0 & f^{\prime}\end{array}\right)$. Then $E A=\left(\begin{array}{cc}c & s+r d \\ 0 & f^{\prime} d\end{array}\right)$. Since $\left\{n^{\prime} e^{\prime} \mid n^{\prime} \in\right.$ $\left.N\left(R_{1}\right), e^{\prime} \in I\left(R_{1}\right)^{\prime}\right\}=N\left(R_{1}\right), c=c_{3} e_{3}$ for some $c_{3} \in N\left(R_{1}\right)$ and $e_{3} \in\{0\} \cup I\left(R_{1}\right)^{\prime}$. So $E A=\left(\begin{array}{cc}c_{3} & s+r d \\ 0 & f^{\prime} d\end{array}\right)\left(\begin{array}{cc}e_{3} & 0 \\ 0 & 1\end{array}\right)$, noting that $\left(\begin{array}{cc}c_{3} & s+r d \\ 0 & f^{\prime} d\end{array}\right) \in N(T)$ and $\left(\begin{array}{cc}e_{3} & 0 \\ 0 & 1\end{array}\right) \in I(T)^{\prime}$.

(iv) Consider the case of $E=\left(\begin{array}{cc}e^{\prime} & r \\ 0 & 1\end{array}\right)$. Then $E A=\left(\begin{array}{cc}e^{\prime} c & e^{\prime} s+r d \\ 0 & d\end{array}\right)$. If $e^{\prime}=0$ then $E A=\left(\begin{array}{cc}0 & r d \\ 0 & d\end{array}\right)=\left(\begin{array}{cc}0 & r d \\ 0 & d\end{array}\right)\left(\begin{array}{ll}0 & 0 \\ 0 & 1\end{array}\right)$, noting that $\left(\begin{array}{cc}0 & r d \\ 0 & d\end{array}\right) \in N(T)$ and $\left(\begin{array}{ll}0 & 0 \\ 0 & 1\end{array}\right) \in I(T)^{\prime}$. Suppose $e^{\prime} \neq 0$. Since $R_{1}$ is right IQNN, $e^{\prime} c=c_{4} e_{4}$ for some $c_{4} \in N\left(R_{1}\right)$ and $e_{4} \in$ $\{0\} \cup I\left(R_{1}\right)^{\prime}$. So $E A=\left(\begin{array}{cc}c_{4} & e^{\prime} s+r d \\ 0 & d\end{array}\right)\left(\begin{array}{cc}e_{4} & 0 \\ 0 & 1\end{array}\right)$, noting that $\left(\begin{array}{cc}c_{4} & e^{\prime} s+r d \\ 0 & d\end{array}\right) \in N(T)$ and $\left(\begin{array}{cc}e_{4} & 0 \\ 0 & 1\end{array}\right) \in I(T)^{\prime}$

Therefore $T$ is a right IQNN ring by (i), (ii), (iii), and (iv).

In the following we consider a method to extend the class of right IQNN rings, by factorizations modulo nil ideals. Recall that an ideal $I$ of a ring $R$ is said to be idempotentlifting if idempotents in $R / I$ can be lifted to $R$. Nil ideals are idempotent-lifting by [16, Proposition 3.6.1]. 
Proposition 3.7. Let $R$ be a ring and $I$ be a nil ideal of $R$. If $R$ is right $I Q N N$ then $R / I$ is right IQNN.

Proof. Suppose that $R$ is right IQNN. Write $\bar{r}=r+I$ with $r \in R$ and $\bar{R}=R / I$. Since $I$ is nil, we have that $I$ is idempotent-lifting by [16, Proposition 3.6.1], $N(\bar{R})=\{\bar{a} \mid a \in N(R)\}$, and $I \cap I(R)^{\prime}=\emptyset$.

Let $\bar{x} \in I(\bar{R})^{\prime}$ and $\bar{a} \in N(\bar{R})$. Then $a \in N(R)$, and there exists $e \in I(R)$ such that $\bar{e}=\bar{x}$. Here $\bar{x} \in I(\bar{R})^{\prime}$ implies $e \in I(R)^{\prime}$. Since $R$ is right IQNN, ex $=b f$ for some $b \in N(R)$ and $f \in I(R)^{\prime}$. Here $\bar{b} \in N(\bar{R})$, and $f \in I(R)^{\prime}$ implies $\bar{f} \neq \overline{0}$ because $I \cap I(R)^{\prime}=\emptyset$. Furthermore, $\bar{f} \neq \overline{1}$ (otherwise, $0 \neq 1-f \in I$, contrary to $I \cap I(R)^{\prime}=\emptyset$ ). Thus $\bar{f} \in I(\bar{R})^{\prime}$ such that $\bar{x} \bar{a}=\bar{e} \bar{a}=\overline{e a}=\overline{b f}=\bar{b} \bar{f}$, showing that $\bar{R}$ is right IQNN.

The condition " $I$ is nil" in Proposition 3.7 cannot be dropped as we see in the following. Let $R=T_{2}(\mathbb{Z})$. Then $R$ is IQNN by Theorem 3.1 or Theorem 3.4. Consider the ideal $I=T_{2}(4 \mathbb{Z})$ of $R$. Then $R / I$ is isomorphic to $T_{2}\left(\mathbb{Z}_{4}\right)$, and $I\left(\mathbb{Z}_{4}\right)=\{0,1\}$. But $\mathbb{Z}_{4}$ is not reduced, and so $T_{2}\left(\mathbb{Z}_{4}\right)$ is not right IQNN by Theorem 3.4. Note that $I$ is not nil. One can see another example in Example 2.6(2).

As a byproduct of the preceding argument, we can conclude that the class of right IQNN rings is not closed under homomorphic images.

The converse of Proposition 3.7 need not hold by the ring $T_{2}(R)$ in Example 3.2, where $R=D_{2}(S)$ over a domain $S . T_{2}(R)$ is not right IQNN, but $T_{2}(R) / I \cong R \oplus R$ is a right IQNN ring by Proposition 2.11(1), where $I=\left(\begin{array}{cc}0 & R \\ 0 & 0\end{array}\right)$ is a nil ideal of $T_{2}(R)$.

From Proposition 3.7, we can obtain an information for matrix rings to be right IQNN.

Corollary 3.8. Let $R$ be a ring and $n \geq 2$. If $D_{n}(R)$ is a right IQNN ring, then so is $R$.

Proof. Suppose that $D_{n}(R)$ is a right IQNN ring. Consider the nilpotent ideal $I=$ $\left\{\left(a_{i j}\right) \in D_{n}(R) \mid a_{i i}=0\right.$ for all $\left.i\right\}$ of $D_{n}(R)$. Then $D_{n}(R) / I \cong R$ is right IQNN by Proposition 3.7.

Following [6], a ring $R$ is called $N R$ if $N(R)$ forms a subring of $R$. NI rings are clearly NR, but the converse need not hold by Antoine [2, Example 4.8]. We first observe the NR property of the rings in Theorem 2.4 and Example 2.5 for our purpose.

Remark 3.9. (1) Let $R=M_{a t}(A)$ be the IQNN ring in Theorem 2.4. Then $R$ is clearly not NR. Note that $R / N^{*}(R) \cong R$ is non-Abelian because $N^{*}(R)=J(R)=0$.

(2) Let $R$ be the IQNN ring in Example 2.5. Then $N(R)=\left\{0, b a+b^{2} a, b a+b^{3} a, b^{2} a+\right.$ $\left.b^{3} a\right\}$. So we have $a N(R)=0, N(R) a=N(R), b N(R)=\left\{0, b^{2} a+b^{3} a\right\}, N(R) b=$ $\left\{0, b^{2} a+b^{3} a\right\}$, and $N(R)^{2}=0$. This implies $N(R)=N^{*}(R)=N_{*}(R)$, and so $R$ is NI (hence NR). Thus $R / N^{*}(R)$ is a reduced ring. Moreover $R / N^{*}(R)$ is a commutative ring, since $a b-b a=a b+b a=b^{2} a+b a \in N^{*}(R)$.

We can construct an NR ring that is neither right IQNN nor NI by help of Theorem 3.4 and [2, Example 4.8] by next example.

Example 3.10. Let $R$ be the ring in [2, Example 4.8], i.e., $R$ is the factor ring of the free algebra $K\langle a, b\rangle$ modulo the ideal generated by $b^{2}$, where $K$ is a field and $a, b$ are noncommuting indeterminates over $K$. Then $R$ is NR by [2, Corollary 3.3], but not NI by the argument in [2, Example 4.8]. Thus $T_{2}(R)$ is NR but not NI by [6, Theorem 2.1] and [11, Proposition 4.(1)], respectively.

We identify $a$ and $b$ with their images in $R$ for simplicity. Assume that $I(R)^{\prime}$ is nonempty. Let $e \in I(R)^{\prime}$. Then $e(1-e)=0=(1-e) e$ with $1-e \in I(R)^{\prime}$. So, by the argument in [12], we get $e \in R b \cap b R$. Then $r b=e=b s$ for some $r, s \in R$, and this yields $0 \neq e=e^{2}=(r b)(b s)=0$ because $b^{2}=0$, a contradiction. Thus $I(R)^{\prime}$ is empty, 
and $I(R)=\{0,1\}$ follows. From this result, we can conclude that $T_{2}(R)$ is neither right nor left IQNN by Theorem 3.4 because $R$ is not reduced.

Based on the example above, we observe a relation between Abelian and NR as follows.

Proposition 3.11. Let $R$ be an $N R$ ring.

(1) If $R$ is non-Abelian then $R[x] / N^{*}(R)[x]$ is an Abelian ring with $N^{*}(R)[x] \neq 0$.

(2) Suppose that $J(R)$ is idempotent-lifting. If $R$ is non-Abelian then $R[x] / J(R)[x]$ is an Abelian ring with $J(R)[x] \neq 0$.

Proof. (1) Since $R$ is NR, $R / N^{*}(R)$ is Abelian by Ster [21, Proposition 3.1]. Let $r \in R$ and $e \in I(R)$. Then $e(r-r e)=e r-e r e=e r(1-e)$ and $(r-e r) e=r e-e r e=(1-e) r e$ are contained in $N(R)$. By the proof of [21, Proposition 3.1], $R($ re-ere) and (er-ere) $R$ are both nil. Here if $R$ is non-Abelian, then there exist $f \in I(R)$ and $s \in R$ such that $f s-f s f \neq 0$ or $s f-f s f \neq 0$. Hence $R(s f-f s f) \neq 0$ or $(f s-f s f) R \neq 0$ by the preceding result. But since $R$ is NR, these are contained in $N^{*}(R)$. This implies $N^{*}(R) \neq 0$, hence $N^{*}(R)[x] \neq 0$. From the isomorphism $R[x] / N^{*}(R)[x] \cong\left(R / N^{*}(R)\right)[x]$, we obtain that $R[x] / N^{*}(R)[x]$ is Abelian by [13, Lemma 8].

(2) It is shown that $R / J(R)$ is an Abelian ring with $J(R) \neq 0$ (hence $J(R)[x] \neq 0$ ), by combining (1) and the results of Chen [4, Propositions 2.2 and 2.16]. Then the result follows the isomorphism $R[x] / J(R)[x] \cong(R / J(R))[x]$ and [13, Lemma 8].

Let $R$ be a ring with $N_{*}(R)=N(R)$. Then $R$ is NR clearly, but we can obtain more strong results related to nilradicals and Jacobson radicals of $R[x]$ as follows. Since $N_{*}(R)=$ $N(R)$, we get $N_{*}(R)=N^{*}(R)=N(R)$; hence $N(R[x])=N^{*}(R[x])=N_{*}(R[x])=$ $N_{*}(R)[x]=N^{*}(R)[x]=N(R)[x]$ by [1, Theorem 3] and [3, Proposition 2.6]. This implies $R[x] / N^{*}(R[x])$ is a reduced ring. By [1, Theorem 1], $J(R[x])=I[x]$ for some nil ideal $I$ of $R$. This entails $J(R[x])=I[x] \subseteq N^{*}(R)[x]=N^{*}(R[x]) \subseteq J(R[x])$ by the preceding argument. So we obtain $J(R[x])=N(R[x])=N^{*}(R[x])=N_{*}(R[x])=N_{*}(R)[x]=$ $N^{*}(R)[x]=N(R)[x]$. Thus $R[x] / J(R[x])$ is also a reduced ring.

However, the preceding argument is not valid for NI rings, even if the rings $R$ with $N_{*}(R)=N(R)$ are obviously NI. In fact, there exist NI rings $R$ in Theorem 3.14 to follow such that $0=N^{*}(R[x]) \subsetneq N^{*}(R)[x] \neq 0$ in $(1)$ and $0=J(R[x]) \subsetneq N^{*}(R)[x]=J(R)[x] \neq 0$ in $(2)$.

The condition " $J(R)$ is idempotent-lifting" in Proposition 3.11(2) is not superfluous as follows.

Example 3.12. Let $S$ be the localization of $\mathbb{Z}$ at the prime ideal $p \mathbb{Z}$, where $p$ is an odd prime; and set $R_{0}$ be the quaternions over $S$. Note $J\left(R_{0}\right)=p R_{0}$ that is the quaternions over $p \mathbb{Z}$. Next set $R=T_{2}\left(R_{0}\right)$. Since $R_{0}$ is a domain and $I\left(R_{0}\right)=\{0,1\}, I(R)=$ $\left\{\left(\begin{array}{ll}0 & 0 \\ 0 & 0\end{array}\right),\left(\begin{array}{ll}1 & 0 \\ 0 & 1\end{array}\right),\left(\begin{array}{ll}1 & a \\ 0 & 0\end{array}\right),\left(\begin{array}{ll}0 & b \\ 0 & 1\end{array}\right) \mid a, b \in R_{0}\right\}$. Note that $R$ is a non-Abelian ring with $J(R)=p R=\left(\begin{array}{cc}p R_{0} & R_{0} \\ 0 & p R_{0}\end{array}\right)$.

We claim that $J(R)$ is not idempotent-lifting. By the argument in [9, Exercise $2 \mathrm{~A}]$, $R_{0} / J\left(R_{0}\right)$ is isomorphic to $\operatorname{Mat}_{2}\left(\mathbb{Z}_{p}\right)$. Hence $R / J(R)$ is isomorphic to $\operatorname{Mat}_{2}\left(\mathbb{Z}_{p}\right) \times \operatorname{Mat}_{2}\left(\mathbb{Z}_{p}\right)$ that is non-Abelian. Here $J(R)$ is not idempotent-lifting. Let $\bar{c}=c+J(R) \mapsto\left(E_{11}, E_{11}\right)$ for $\left(E_{11}, E_{11}\right) \in \operatorname{Mat}_{2}\left(\mathbb{Z}_{p}\right) \times \operatorname{Mat}_{2}\left(\mathbb{Z}_{p}\right)$. Then $\bar{c} \in I(R / J(R))^{\prime}$ because $\left(E_{11}, E_{11}\right) \in$ $I\left(\operatorname{Mat}_{2}\left(\mathbb{Z}_{p}\right) \times \operatorname{Mat}_{2}\left(\mathbb{Z}_{p}\right)\right)^{\prime}$. Moreover $\bar{c}=\left(\begin{array}{cc}c_{1} & d \\ 0 & c_{2}\end{array}\right)+J(R)$ with $c_{i} \notin p R_{0}$. Assume that there exists $e \in I(R)$ such that $\bar{c}=\bar{e}$. Since $\bar{c} \mapsto\left(E_{11}, E_{11}\right)$, e must be 1 because $I(R)=\left\{\left(\begin{array}{ll}0 & 0 \\ 0 & 0\end{array}\right),\left(\begin{array}{ll}1 & 0 \\ 0 & 1\end{array}\right),\left(\begin{array}{ll}1 & a \\ 0 & 0\end{array}\right),\left(\begin{array}{ll}0 & b \\ 0 & 1\end{array}\right) \mid a, b \in R_{0}\right\}$. This implies $1-c \in J(R)$, hence $c$ is a unit, a contradiction. Thus $\bar{c}$ cannot be lifted to an idempotent in $R$. 
We next consider the right IQNN property of polynomial rings. Recall that Köthe's conjecture means that the sum of two nil right ideals is nil. In the following we see two kinds of Abelian polynomial rings which is not NI. To see that, we need to observe a property of the nil ring constructed by Smoktunowicz [20].

Example 3.13. Let $K$ be any countable field. Then, by [20, Theorem 12], there is a nil algebra $R_{0}$ over $K$ generated by three elements (say $a, b, c$ ), such that $R_{0}[x, y]$ is not nil, where $R_{0}[x, y]$ is the polynomial ring with commuting indeterminates $x, y$ over $R_{0}$. Set $R=K+R_{0}$. Then $N(R)=R_{0}=N^{*}(R)$ because $R / R_{0} \cong K$, concluding that $R$ is an NI ring. Moreover $R_{0}[x, y]$ being non-nil implies $R[x, y]$ being non-NI, because $a+b x+c y$ is not nilpotent by the proof of [20, Theorem 12], in spite of $a, b x, c y \in N(R[x, y])$.

We claim that $N_{*}(R) \subsetneq N^{*}(R)$. Assume $N_{*}(R)=N^{*}(R)$. Since $a, b, c \in N^{*}(R)$, we have $a, b, c \in N_{*}(R)$. Hence $a+b x+c y \in N_{*}(R[x, y])$ because $N_{*}(R)[x, y]=N_{*}(R[x])[y]=$ $N_{*}(R[x, y])$ by [1, Theorem 3]. This is contrary to $a+b x+c y \notin N(R[x, y])$. Thus $N_{*}(R) \subsetneq N^{*}(R)$.

The following is based on the construction of Smoktunowicz and argument in Example 3.13 .

Theorem 3.14. (1) Given any countable field $K$, there exists a semiprime non-reduced NI algebra $R$ over $K$ such that $N^{*}(R[x])=0$ and $R[x]$ is Abelian but not NI.

(2) If Köthe's conjecture has a negative solution, then there exists a semiprime nonreduced NI ring $R$ such that $J(R[x])=0$ and $R[x]$ is Abelian but not $N I$.

Proof. (1) By [4, Theorem 3.8], there exists a nil algebra $S$ over $K$ such that $N^{*}(S[x])=0$. Set $R=K+S$. Then $N(R)=S=N^{*}(R)=J(R)$ and so $R$ is NI. Furthermore, $R$ is Abelian because $R / J(R) \cong K$ (hence $R$ is local). Then $R[x]$ is also Abelian by [13, Lemma 8]. We can assume that $R$ is semiprime through the isomorphism $\frac{R}{N^{*}(R)} \cong \frac{R}{N_{*}(R)} / \frac{N^{*}(R)}{N_{*}(R)}$.

From the isomorphism $R[x] / S[x]=R[x] / N^{*}(R)[x] \cong\left(R / N^{*}(R)\right)[x]=(R / S)[x] \cong$ $K[x]$, we have $N^{*}(R[x]) \subseteq N(R[x]) \subseteq S[x]$. This implies that

$$
N(R[x])=N(R[x]) \cap S[x]=N(S[x]) \text { and } N^{*}(R[x])=N^{*}(R[x]) \cap S[x] \subseteq N^{*}(S[x]),
$$

noting that $N^{*}(R[x])$ is a nil ideal of $S[x]$. But $N^{*}(S[x])=0$ and $N^{*}(R[x])=0$ follows. Every monomial in $S[x]$ is nilpotent, and so $0 \neq N(S[x]) \subseteq R[x]$. Hence $R[x]$ is not NI.

(2) Krempa [15] proved that Köthe's conjecture is equivalent to the problem of whether for each nil algebra $S$ over a field, the polynomial ring over $S$ is Jacobson radical.

Suppose that Köthe's conjecture has a negative solution. Then, by Chen [4, Lemma 3.6], there exists a nil algebra $S$ over some countable field $K$ such that $J(S[x])=0$. Chen obtained this result, based on the construction of Smoktunowicz [20] and the preceding fact of Krempa.

Next set $R=K+S$ and consider $R[x]$. Then $R[x]$ is Abelian by the argument in (1). We can assume that $R$ is semiprime through the isomorphism $\frac{R}{J(R)} \cong \frac{R}{N_{*}(R)} / \frac{J(R)}{N_{*}(R)}$. By [1, Theorem 1], $J(R[x])=I[x]$ for some nil ideal $I$ of $R$. But $I$ is contained in $S$ by a similar method to (3). This implies $J(R[x])=S[x] \cap J(R[x])=J(S[x])=0$. Since $R[x]$ is not reduced, $N^{*}(R[x]) \subseteq J(R[x])=0$ implies that $R[x]$ is not NI.

The following shows that the condition "a ring $R$ being right IQNN" is necessary for the condition " $R[x]$ being right IQNN".

Proposition 3.15. Let $R$ be a ring.

(1) Let $f(x)=\sum_{i=0}^{m} a_{i} x^{i} \in I(R[x])$. If $a_{0}$ is central, then $f(x)=a_{0}$.

(2) If $f(x)=\sum_{i=0}^{m} a_{i} x^{i} \in I(R[x])^{\prime}$, then $a_{0} \in I(R)^{\prime}$.

(3) If $R[x]$ is a right IQNN ring, then so is $R$.

(4) If $R$ is a reduced ring then $T_{n}(R)[x]$ is an IQNN ring for all $n \geq 2$. 
Proof. (1) This is shown by the proof of [13, Lemma 8].

(2) Let $f(x)=\sum_{i=0}^{m} a_{i} x^{i} \in I(R[x])^{\prime}$. Then $a_{0} \neq 0$ obviously. Assume $a_{0}=1$. Then $f(x)=1$ by $(1)$, contrary to $f(x) \notin I(R[x])^{\prime}$. Therefore $a_{0} \in I(R)^{\prime}$.

(3) Assume that $R[x]$ is right IQNN. Let $a \in N(R)$ and $e \in I(R)^{\prime}$. Since $R[x]$ is right IQNN, there exist $g(x)=\sum_{j=0}^{n} b_{j} x^{j} \in N(R[x])$ and $f(x)=\sum_{i=0}^{m} a_{i} x^{i} \in I(R[x])^{\prime}$ such that $e a=g(x) f(x)$. Then $b_{0} \in N(R)$ clearly, and $a_{0} \in I(R)^{\prime}$ by (1). Thus $e a=b_{0} a_{0}$ and so $R$ is right IQNN.

(4) Let $R$ be a reduced ring. Then $T_{n}(R[x])$ is IQNN by Theorem 3.1 since $R[x]$ is reduced. Moreover $T_{n}(R)[x] \cong T_{n}(R[x])$ implies that $T_{n}(R)[x]$ is IQNN.

Finally, regarding Theorem 2.4, we raise the following question.

Question. Is $\mathrm{Mat}_{2}(A)$ a right IQNN ring over a commutative domain $A$ ?

Acknowledgment. The authors thank the referees deeply for very careful reading of the manuscript and valuable suggestions in depth that improved the paper by much. The first author was supported by Basic Science Research Program through the National Research Foundation of Korea(NRF) funded by the Ministry of Education (2016R1D1A1B03931190) and the third named author was supported by the National Natural Science Foundation of China (11361063).

\section{References}

[1] S.A. Amitsur, Radicals of polynomial rings, Canad. J. Math. 8, 355-361, 1956.

[2] R. Antoine, Nilpotent elements and Armendariz rings, J. Algebra 319, 3128-3140, 2008.

[3] G.F. Birkenmeier, H.E. Heatherly, and E.K. Lee, Completely prime ideals and associated radicals, in: Proc. Biennial Ohio State-Denison Conference 1992, edited by S.K. Jain and S.T. Rizvi, World Scientific, Singapore-New Jersey-London-Hong Kong, 102-129, 1993.

[4] W. Chen, On linearly weak Armendariz rings, J. Pure Appl. Algebra 219, 1122-1130, 2015.

[5] E.-K. Cho, T.K. Kwak, Y. Lee, Z. Piao, and Y. Seo, A structure of noncentral idempotents, Bull. Korean Math. Soc. 55, 25-40, 2018.

[6] Y. Chun, Y. C. Jeon, S. Kang, K. N. Lee, Y. Lee, A concept unifying the Armendariz and NI conditions, Bull. Korean Math. Soc. 48, 115-127, 2011.

[7] E.H. Feller, Properties of primary noncommutative rings, Trans. Amer. Math. Soc. 89, 79-91, 1958.

[8] K.R. Goodearl, Von Neumann Regular Rings, Pitman, London 1979.

[9] K.R. Goodearl and Jr. R.B. Warfield, An Introduction to Noncommutative Noetherian Rings, Cambridge University Press, Cambridge-New York-Port Chester-MelbourneSydney, 1989.

[10] C. Huh, H.K. Kim, and Y. Lee, p.p. rings and generalized p.p. rings, J. Pure Appl. Algebra 167, 37-52, 2002.

[11] S.U. Hwang, Y.C. Jeon, and Y. Lee, Structure and topological conditions of NI rings, J. Algebra 302, 186-199, 2006.

[12] H.K. Kim, T.K. Kwak, Y. Lee, and Y. Seo, Corrigendum Insertion of units at zero products [J. Algebra Appl. Vol. 17, 1850043 (20 pp), 2018], J. Algebra Appl. Vol. 17, 1892002, 2pp, 2018.

[13] N.K. Kim and Y. Lee, Armendariz rings and reduced rings, J. Algebra 223, 477-488, 2018.

[14] N.K. Kim, Y. Lee, and Y. Seo, Structure of idempotents in rings without identity, J. Korean Math. Soc. 51, 751-771, 2014. 
[15] J. Krempa, Logical connections among some open problems concerning nil rings, Fund. Math. 76, 121-130, 1972.

[16] J. Lambek, Lectures on Rings and Modules, Blaisdell Publishing Company, Waltham, 1966.

[17] G. Marks, On 2-primal Ore extensions, Comm. Algebra 29, 2113-2123, 2001.

[18] N.H. McCoy, Generalized regular rings, Bull. Amer. Math. Soc. 45, 175-178, 1939.

[19] M. Ôhori, On strongly $\pi$-regular rings and periodic rings, Math. J. Okayama Univ. 27, 49-52, 1985.

[20] A. Smoktunowicz, Polynomial rings over nil rings need not be nil, J. Algebra 233, 427-436, 2000.

[21] J. Ster, Rings in which nilpotents form a subring, Carpathian J. Math. 32, 251-258, 2016. 